\title{
Non-Viral Фc31 Integrase Mediated In Vivo Gene Delivery to the Adult Murine Kidney
}

\author{
Daniel C Chung ${ }^{1}$, Matthew C Canver ${ }^{1}$, Xiaofeng Zuo ${ }^{2}$ and Jean Bennett and Joshua H Lipschutz ${ }^{2,3 *}$ \\ ${ }^{1}$ FM Kirby Center of Molecular Ophthalmology, Scheie Eye Institute, USA \\ ${ }^{2}$ Department of Medicine, Medical University of South Carolina, USA \\ ${ }^{3}$ Department of Medicine, Ralph H Johnson VAMC, USA
}

*Corresponding author: Joshua H Lipschutz, Medical University of South Carolina, Clinical Science Building 829,96 Jonathan Lucas Street, Charleston, SC 29425, USA

Submission: 侮January 23, 2018; Published: 每 February 05, 2018

\begin{abstract}
Background/Aims: Degeneration of primary cilia has been implicated in many diseases, including retinal degeneration and polycystic kidney disease. This study aims to demonstrate gene transfer to adult murine ciliated kidney tubule cells, in vivo, facilitated by $\varphi \mathrm{C} 31$ integrase and electroporation.

Methods: Cohorts of 6-10 week old adult C57BL/6 mice were used. After adequate anesthesia via inhaled isoflurane, mice were prepped and draped for abdominal surgery. A midline laparotomy was performed, and the left kidney was isolated. Connective and adipose tissue was bluntly dissected away from the ureter and renal pelvis. A $10 \mu \mathrm{L}$ retrograde intra-ureteral microinjection was performed utilizing a heat-pulled glass capillary tube. The capillary tube was filled with two plasmids, one containing the marker gene, enhanced green fluorescent protein with the attachment site B sequence (pCMV.eGFP.attB), and the other encoding for the integrase enzyme (pCMV.Int), and electroporation was performed. Three days later, the mice were sacrificed, and kidneys were collected, placed in 4\% paraformaldehyde, cryoprotected, sectioned, and evaluated using fluorescence microscopy.
\end{abstract}

Results: Green fluorescence protein expression was detected in renal papilla and renal tubule cells. No gross inflammation was appreciated.

Conclusion: $\varphi \mathrm{C}$ integrase can facilitate gene transfer to the adult murine kidney in vivo. Further optimization will be needed to increase efficiency of transduction, document stability of transgene expression, and evaluate potential toxicities.

Keywords: Gene therapy; Kidney; Mouse

Abbreviations: PKD: Polycystic Kidney Disease; AKI: Acute Kidney Injury; ESRD: End-Stage Renal Disease

\section{Introduction}

Gene therapy involves delivery and targeting of exogenous DNA into cells to provide a therapeutic protein. Though no renal disease has yet been successfully treated in animals or patients using gene therapy, this modality holds great promise [1]. Ideally, a gene therapy vector should be non-toxic, non-immunogenic, easy to produce, and efficient in protecting and delivering DNA into the target cells. Exogenous DNA can be delivered with non-viral or viral vectors, each offering advantages and disadvantages. The use of viruses ensures highly efficient gene transfer to mammalian cells, as viruses have evolved specialized mechanisms for cell binding and intracellular delivery of DNA. Some viruses, however, such as adenovirus, can evoke severe and even fatal immunogenic responses [2]. Retroviruses and lentiviruses suffer from random integration, and have a tendency to integrate near transcriptionally active genes resulting in leukemia-like syndromes in recipients [3]. Non-viral vectors have low toxicity, and low immunogenicity, but also lower efficiency, and transient expression currently limits their use as a therapeutic gene therapy vector [4]. Primary cilia are present in almost all vertebrate cell types. Degeneration of primary cilia has been implicated in many diseases, including retinal degeneration and polycystic kidney disease (PKD). Gene targeting in other paradigms such as retinal photoreceptor outer segments (cilia) has been widely demonstrated $[5,6]$. This study investigates non-viral $\varphi \mathrm{C} 31$ integrase mediated transfection [7] of the primary ciliated renal collecting tubule cells.

Streptomyces phage $\varphi \mathrm{C} 31$ integrase functions through sitespecific recombination between sites on the phage and bacterial genomes. These specific sites are termed $a t t P$ and $a t t B$, respectively [8]. $\varphi \mathrm{C} 31$ integrase-mediated gene delivery has the advantages of insertion of DNA sequences of greater than $10 \mathrm{~kb}$, and it does not pose immunogenic and bio safety risks that are associated with viral vectors. The kidney is accessible to gene delivery by different routes including via the renal artery, injection into the parenchyma, and retrograde via the ureter. Retrograde injection is an attractive route for treating a variety of diseases and disorders affecting renal tubule cells, as these cells are readily accessible in patients 
by retrograde ureteral injection using a ureteroscope. Equally important, retrograde ureteral injection further minimizes the potential for a toxic immunologic reaction. We demonstrate here the feasibility of transfecting renal tubule cells with $\varphi \mathrm{C}$ integrase and electroporation.

\section{Materials and Methods}

\section{Plasmid vector}

A plasmid vector, pCMV.eGFP.attB, contained the marker gene (eGFP) with the attachment site B sequence. A second vector, pCMV. Int, encodes for the integrase enzyme (a gift from Michelle Calos, PhD, Stanford University, Palo Alto, CA).

\section{Surgical technique}

Animals were placed under adequate inhaled isoflurane anesthesia (3\% for induction, $2 \%$ for maintenance) and were placed supine on a platform beneath a dissecting microscope. The surgical area was prepped with alcohol swabs followed by betadine topical antiseptic. Ventral lateral laparotomy was performed and the left kidney was isolated. Connective and adipose tissues were bluntly dissected away from the ureter and renal pelvis. A square knot was tied around the distal ureter to prevent flow of injection contents to the bladder. $10 \mu \mathrm{L}$ retrograde intra-ureteral microinjection was performed utilizing a heat-pulled glass capillary tube. Tweezertype electrodes were placed around the injected kidney and it was electroporated using high-voltage, short duration pulses (voltage was set to $100 \mathrm{~V}$ and five pulses of length $0.005 \mathrm{~ms}$ were applied) The square knot was removed from the distal ureter post-injection. Muscle and skin incisions were closed separately with a running 7-0 silk suture. Adult (6-10 week old) C57BL/6 mice were used for all studies. All experiments were performed in compliance with institutional regulations and were approved by the Institutional Animal Care and Use Committee at the University of Pennsylvania and the Medical University of South Carolina.

\section{Experimental design}

Two cohorts of five adult (6-10 week old) C57BL/6 wild type mice were injected with pCMV.eGFP.attB and pCMV.Int. Contralateral kidneys were uninjected and used as controls.

\section{Histology}

The kidneys were removed following carbon dioxide-induced euthanasia and were then fixed in a solution containing $4 \%$ paraformaldehyde and PBS. The kidneys were then cryoprotected in a solution containing 30\% sucrose and PBS overnight, whereupon the kidneys were then frozen in optimal cutting temperature compound (purchased from Fisher Scientific, Pittsburgh, PA, USA). For each kidney, serial sections were cut using a cryostat (made by Reichert Jung model 822; Leica Microsystems, Inc., Wetzlar, Germany), and counterstained with 4', 6-diamidino-2phenylindole,dihydrochloride, more commonly known as DAPI, Vectashield mounting medium (made by Vector Labs, Burlingame, CA, USA). All slides were then mounted and imaged using a Leica DME microscope (made by Leica Microsystems, Inc., Buffalo Grove,
IL, USA), that was equipped with epifluorescence capability. The images were then captured using a Hamamatsu digital camera, and were processed with Openlab 2.2 image analysis software (made by Improvision, Inc., Boston, MA, USA) as previously described [9].

Results

\section{Animal preparation}

Two cohorts of five 6-10 week old adult C57BL/ 6 mice under inhaled isoflurane anesthesia were placed supine on a platform beneath a dissecting microscope. Ventral lateral laparotomy was performed and the left kidney was isolated. Connective tissue was bluntly dissected away from the ureter and renal pelvis. A square knot was tied around the distal ureter to prevent backflow of injection contents.

\section{Transfection of kidney tubule cells}

Plasmid vectors, pCMV.eGFP.attB, which contained the marker gene (eGFP) with the attachment site B sequence, and pCMV.Int, which encodes the integrase enzyme were used. $10 \mu \mathrm{L}$ retrograde intra-ureteral microinjection was performed utilizing a pulled glass capillary tube. Tweezer-type electrodes were placed around the injected kidney and it was electroporated using high-voltage, short duration pulses (voltage was set to $100 \mathrm{~V}$ and five pulses of length $0.005 \mathrm{~ms}$ were applied). The square knot was removed from the distal ureter post-injection.

\section{GFP reporter expression}

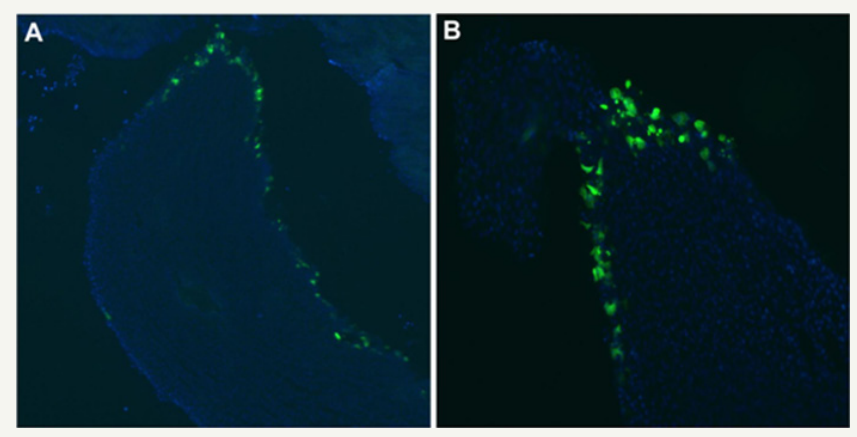

Figure 1: Efficient Transduction of Epithelial Cells in the Renal Papilla.

A,B) Three days following injection of pCMV.eGFP.attB/ pCMV.Int and electroporation, kidneys were removed following carbon dioxide-induced euthanasia and immediately fixed in $4 \%$ paraformaldehyde/PBS. They were cryoprotected in $30 \%$ sucrose/PBS overnight and frozen. For each kidney, serial sections were cut with a cryostat. eGFP expression (green) was seen in the renal papilla after intra-ureteral injection of pCMV.eGFP.i/pCMV.Int. The cell nuclei are stained with DAPI (blue).

Three days post transduction, kidneys were removed following carbon dioxide-induced euthanasia and immediately fixed in $4 \%$ paraformaldehyde/PBS. The kidneys were cryoprotected in $30 \%$ sucrose/PBS overnight and frozen. For each kidney, serial sections were cut with a cryostat. All were mounted and analyzed with epifluorescence, and images were captured. eGFP expression was seen in the renal papilla after intra-ureteral injection of pCMV.eGFP. 


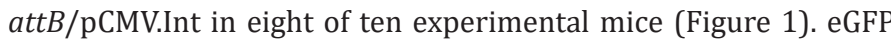
expression was clearly seen in the renal tubule cells after intra ureteral injection of pCMV.eGFP.attB/pCMV.Int (Figure 2). No gross inflammation or cell damage was appreciated, nor GFP fluorescence seen in the untreated kidney or other organs (data not shown).

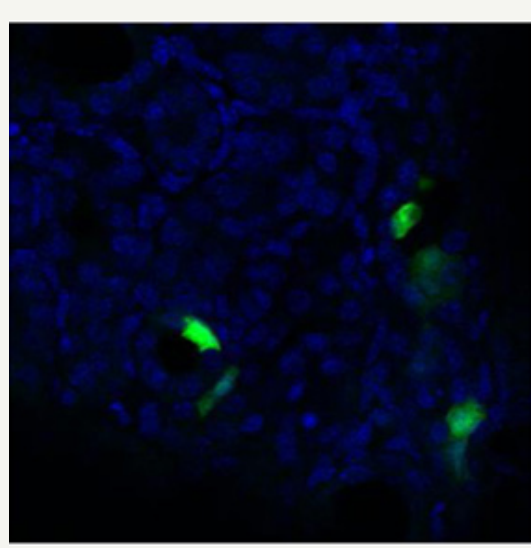

Figure 2: Transduction of Epithelial Renal Tubule Cells Three days following injection of pCMV.eGFP.attB/pCMV. Int and electroporation, kidneys were removed following carbon dioxide-induced euthanasia and immediately fixed in $4 \%$ paraformaldehyde/PBS. They were cryoprotected in $30 \%$ sucrose/PBS overnight and frozen. For each kidney, serial sections were cut with a cryostat. eGFP expression (green) was seen in the renal tubule cells after intra-ureteral injection of pCMV.eGFP. attB/pCMV.Int. The cell nuclei are stained with DAPI (blue).

\section{Discussion}

We report two principal findings. First, we show excellent transduction efficiency of renal tubule cells using $\varphi$ C31 integrase mediated transfection with electroporation. Electroporation of plasmid DNA has been used in embryonic kidneys [10], and some adult models [11], though not, to our knowledge, in combination with $\varphi$ C31 integrase, which greatly enhances transduction efficiency. Our second principle finding is that we can specifically and efficiently deliver plasmid DNA to renal tubule epithelial cells via a modified retrograde approach. While, retrograde injection of the ureter has been used to introduce reporter genes $[12,13]$ and AAV [14], transduction efficiencies have not been optimal. An obvious advantage to a retrograde approach is that, if successful in animal trials of renal tubular disease, it could be scaled up to humans, and pCMV.eGFP.attB/pCMV.Int encoding the appropriate cargo could be injected in a minimally invasive manner by urologists using ureteroscopy, with or without electroporation via laparoscopy. Since many kidney diseases are bilaterally symmetrical, it is possible to perform controlled experiments targeting renal disease by using one kidney as the experimental kidney and the contralateral kidney as the control. In summary, $\varphi \mathrm{C} 31$ integrase can facilitate gene transfer to the adult murine kidney in vivo. $\varphi$ C31 integrase mediated gene delivery has been demonstrated to be effective in other paradigms, and may prove to be an effective method for non-viral gene delivery to renal tissues. Further optimization, however, will be needed to increase efficiency of transduction, document stability of transgene expression, and evaluate potential toxicities. If successful in humans, gene delivery using $\varphi$ C31 integrase, electroporation, and plasmid DNA injected in a retrograde manner would represent a novel treatment for the many disorders that manifest in renal tubule epithelial cells. Retrograde injection could be performed relatively non-invasively by Urologists using an ureteroscope. Electroporation could be then accomplished in a laparoscopic manner. This is especially important given that there are currently no approved treatments, other than supportive care, for renal tubular disorders such as ADPKD and acute kidney injury (AKI). Moreover, as ADPKD does not result in end-stage renal disease (ESRD) until the fifth or sixth decade of life, 50\% transduction efficiency, or even less, could make ADPKD a disease that does not result in ESRD within a normal lifespan.

\section{References}

1. Worgall S (2005) A realistic chance for gene therapy in the near future. Pediatr Nephrol 20(2): 118-124.

2. Raper SE, Chirmule N, Lee FS, Wivel NA, Bagg A, et al. (2003) Fatal systemic inflammatory response syndrome in a ornithine transcarbamylase deficient patient following adenoviral gene transfer. Molecular genetics and metabolism 80(1-2): 148-158.

3. Hacein BAS, Von KC, Schmidt M, McCormack MP, Wulffraat N, et al. (2003) LMO2-associated clonal T cell proliferation in two patients after gene therapy for SCID-X1. Science 302 (5644): 415-419.

4. Tomasoni S, Benigni A (2004) Gene therapy: how to target the kidney. Promises and pitfalls. Curr Gene Ther 4(11): 115-122.

5. Maguire AM, High KA, Auricchio A, Wright JF, Pierce EA (2009) Agedependent effects of RPE65 gene therapy for Leber's congenital amaurosis: a phase 1 dose-escalation trial. Lancet 374(9701): 15971605.

6. Maguire AM, Simonelli F, Pierce EA, Pugh EN, Mingozzi F, et al. (2008) Safety and efficacy of gene transfer for Leber's congenital amaurosis. N Engl J Med 358(21): 2240-2248.

7. Chalberg TW, Genise HL, Vollrath D, Calos MP (2005) phiC31 integrase confers genomic integration and long-term transgene expression in rat retina. Invest Ophthalmol Vis Sci 46(6): 2140-2146.

8. Groth AC, Olivares EC, Thyagarajan B, Calos MP (2000) A phage integrase directs efficient site-specific integration in human cells. Proc Natl Acad Sci USA 97(11): 5995-6000

9. Lebherz C, Maguire A, Tang W, Bennett J, Wilson JM (2008) Novel AAV serotypes for improved ocular gene transfer. J Gene Med 10(4): 375-382.

10. Alie TM, Vrljicak PJ, Myburgh DB, Gupta IR (2007) Microinjection and electroporation of embryonic kidney explants: an improved method. Kidney Int 72(1): 121-125.

11. Brown PA, Bodles-BAM, Pope MA, Draghia AR (2009) Gene therapy by electroporation for the treatment of chronic renal failure in companion animals. BMC biotechnology 9: 4 .

12. Gusella GL, Fedorova E, Hanss B, Marras D, Klotman ME, et al. (2002) Lentiviral gene transduction of kidney. Hum Gene Ther 13(3): 407-414.

13. Moullier P, Friedlander G, Calise D, Ronco P, Perricaudet M, et al. (1994) Adenoviral- mediated gene transfer to renal tubular cells in vivo. Kidney Int 45(4): 1220-1225.

14. Chung DC, Fogelgren B, Park KM, Heidenberg J, Zuo X, et al. (2011) Adeno-associated virus-mediated gene transfer to renal tubule cells via a retrograde ureteral approach. Nephron Extra 1(1): 217-223. 
Creative Commons Attribution 4.0 International License

For possible submission use the below is the URL
Your subsequent submission with Crimson Publishers will attain the below benefits

- High-level peer review and editorial services

- Freely accessible online immediately upon publication

- Authors retain the copyright to their work

- Licensing it under a Creative Commons license

- Visibility through different online platforms

- Global attainment for your research

- Article availability in different formats (Pdf, E-pub, Full Text)

- Endless customer service

- Reasonable Membership services

- Reprints availability upon request

- One step article tracking system 